\title{
PD-1 deficiency protects experimental colitis via alteration of gut microbiota
}

\author{
Seong Jeong Park ${ }^{1}$, Ji-Hae Kim ${ }^{1}$, Mi-Young Song ${ }^{2}$, Young Chul Sung ${ }^{1,2}$, Seung-Woo Lee ${ }^{1,2, *} \mathcal{E}$ Yunji Park $^{2, *}$ \\ ${ }^{1}$ Department of Life Sciences, ${ }^{2}$ Division of Integrative Biosciences and Biotechnology, Pohang University of Science and Technology, \\ Pohang 37673, Korea
}

Programmed cell death-1 (PD-1) is a coinhibitory molecule and plays a pivotal role in immune regulation. Here, we demonstrate a role for PD-1 in pathogenesis of inflammatory bowel disease (IBD). Wild-type (WT) mice had severe wasting disease during experimentally induced colitis, while mice deficient for PD-1 (PD-1 ${ }^{-/}$) did not develop colon inflammation. Interestingly, PD-1 ${ }^{-1-}$ mice cohoused with WT mice became susceptible to colitis, suggesting that resistance of PD-1 ${ }^{-1-}$ mice to colitis is dependent on their gut microbiota. $16 S$ rRNA gene-pyrosequencing analysis showed that PD-1 ${ }^{-1-}$ mice had altered composition of gut microbiota with significant reduction in Rikenellaceae family. These altered colon bacteria of PD-1 ${ }^{-1-}$ mice induced less amount of inflammatory mediators from colon epithelial cells, including interleukin (IL)-6, and inflammatory chemokines. Taken together, our study indicates that PD-1 expression is involved in the resistance to experimental colitis through altered bacterial communities of colon. [BMB Reports 2017; 50(11): 578-583]

\section{INTRODUCTION}

The gastrointestinal tract of mammals and vertebrates is colonized by trillions of microorganisms that co-exist peacefully with the host, helping dietary digestion and protecting the host against rapid colonization of pathogens (1). Studies using germ-free animals have revealed that commensal microbiota is essential for development and function of competent immune systems in both periphery and intestinal mucosa $(1,2)$. When the acquisition or compositions of gut microbiota, however, are altered by genetic, environmental, or other unknown

*Corresponding authors. Yunji Park, Tel: +82-54-279-0658; Fax: +82-54-279-0657; E-mail: yunji@postech.ac.kr, Seung-Woo Lee, Tel: +82-54-279-2355; Fax: +82-54-279-0657; E-mail: sw_lee@ postech.ac.kr

https://doi.org/10.5483/BMBRep.2017.50.11.165

Received 23 August 2017, Revised 7 September 2017, Accepted 22 September 2017

Keywords: Colitis, Inflammation, Metagenomics, Microbiota, PD-1 factors, these organisms are able to drive gut inflammation, leading to inflammatory bowel disease (IBD) (3). IBD is chronic inflammatory condition of the gastrointestinal tract, including Crohn's disease (CD) and ulcerative colitis (UC). Although the precise pathogenesis of IBD has not been clearly elucidated, recent evidence has revealed that the aberrant immune activation against gut microbiota contributes to the pathogenesis of $\operatorname{IBD}(4,5)$. In line with this, composition of gut microbiota is altered or abnormal in IBD patients (6-8).

Several lines of evidence indicate that $T$ cells activated abnormally in the intestine are pathogenic effectors in IBD progress, resulting in secretion of proinflammatory cytokines, recruiting inflammatory cells, and gut injury $(5,9)$. Therefore, therapeutic strategies targeting $\mathrm{T}$ cell signaling, such as CTLA-4, have been evaluated to prevent activation of pathogenic mucosal $T$ cells in IBD (10). In addition to CTLA-4, programmed death-1 (PD-1) is a negative costimulatory molecule, expressed on activated T/B cells and monocytes and exerts inhibitory functions via engagement with its ligand, PD-L1 $(11,12)$. In recent years, PD-1 has been studied for its regulatory function in T cell activation, immune tolerance, and autoimmunity, and considered as a potent therapeutic target for several autoimmune diseases (reviewed in ref. 13). Only few studies, however, report the role of PD-1/PD-L1 in intestinal inflammation $(14,15)$. An early study reports that PD-1 is highly expressed on $T$ cells in inflamed colon from both IBD patients and colitic mice, and blockade of PD-L1 suppressed experimental colitis (14). Conversely, a recent study demonstrates gut epithelium-expressed PD-L1 as a key regulator of intestinal inflammation by inhibiting innate immune cells (15). Nevertheless, the role of PD-1/PD-L1 pathway in the pathogenesis of IBD and the underlying mechanisms have not been extensively explored and remains largely unknown. In this study, we reveal that PD-1 deficiency alters composition of gut microbiota, thereby suppressing development of experimentally induced colitis.

\section{RESULTS AND DISCUSSION}

PD-1-deficient mice are less susceptible to DSS-induced colitis

To investigate the involvement of PD-1 in development of

ISSN: 1976-670X (electronic edition)

Copyright (C) 2017 by the The Korean Society for Biochemistry and Molecular Biology

(c) This is an open-access article distributed under the terms of the Creative Commons Attribution Non-Commercial License (http://creativecommons.org/licenses/by-nc/4.0) which permits unrestricted non-commercial use, distribution, and reproduction in any medium, provided the original work is properly cited. 
colitis, we employed dextran sodium sulfate (DSS)-induced murine colitis model that mimics clinical and histological features of human IBD (16). Wild type (WT) and PD-1deficient $\left(\mathrm{PD}-1^{-/}\right)$mice were fed with $2 \%$ DSS in drinking water for $9 \mathrm{~d}$, and parameters for colitis evaluation were determined. WT mice given $2 \%$ DSS showed time-dependent body weight loss $(82.3 \pm 2.2 \%$ of original weight at day 9$)$, whereas $\mathrm{PD}-1^{-1-}$ mice rarely lost their weight during DSS administration for $9 \mathrm{~d}(95.7 \pm 0.9 \%$ of original weight at day 9) (Fig. 1A). Concomitantly, colon shortening was not significantly observed in DSS-treated PD- $1^{-1-}$ mice, compared to DSS-fed WT mice (Fig. 1B). Histological analysis of colon from DSS-treated WT mice showed severe inflammation and infiltration of inflammatory cells, while the histological scores were significantly improved in DSS-fed PD- $1^{-1-}$ mice (Fig. 1C).

In addition to its inhibitory effect on T cells, PD-1 negatively regulates the function of innate immune cells, as shown by down-regulation of cytokine production from macrophages upon PD-1 engagement (12) and elevated production of interleukin (IL)-12 from PD-1 ${ }^{-1-}$ dendritic cells (DC) (17). Consistently, we observed higher IL-12p40 production in

A

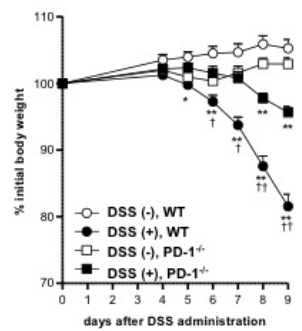

$$
\text { c }
$$
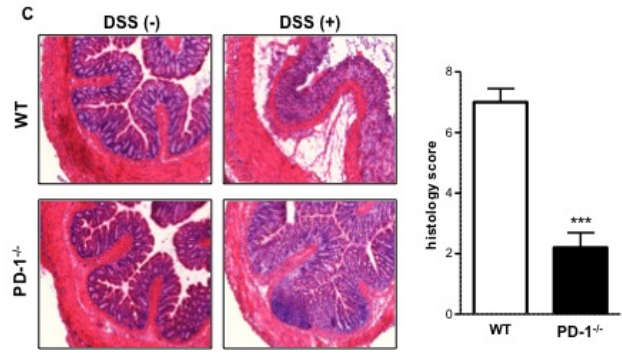

Fig. 1. PD-1 ${ }^{-1-}$ mice are resistant to DSS-induced colitis. WT and PD-1 ${ }^{-1-}$ mice were given $2 \%(\mathrm{w} / \mathrm{v})$ DSS in drinking water (DSS positive) or sterilized tap water (DSS negative) as a control. (A) Change in body weight ( $\%$ of initial body weight, mean \pm SEM) during DSS-administration. $* P<0.05, * * P<0.01$ vs. DSS (-), WT mice; ${ }^{\dagger} \mathrm{P}<0.05,{ }^{+} \mathrm{P}<0.01$ vs. DSS $(+), \mathrm{PD}^{+} 1^{-1-}$ mice by Student's t-test. Data are pooled from three independent experiments. (B) Colon shortening of WT or PD-1 ${ }^{-1-}$ mice at $9 \mathrm{~d}$ after DSS-treatment. Each dot in the graph represents a single mouse. ${ }^{* * P}<0.01$ by Student's $t$-test. Data are pooled from two different experiments. (C) Histology of colons at $9 \mathrm{~d}(\times 400)$. Histology score of DSS-treated WT $(n=5)$ and PD-1 ${ }^{-1-}$ mice $(n$ $=5$ ) were shown in the bar graph (mean \pm SEM). $* * * P<$ 0.0001 vs. WT controls by Student's $t$-test. laminar propria mononuclear cells (LPMC) from colon of both naïve and DSS-treated PD-1 ${ }^{-1-}$ mice, as compared to the WT control (Supplementary Fig. 1A). We previously showed the protective effect of IL-12p40 on DSS-induced colitis (18), thus we next examined whether higher production of IL-12p40 in $\mathrm{PD}-1^{-1-}$ mice is critical for decreased incidence of colitis in $\mathrm{PD}-1^{-1-}$ mice. We observed that treatment of neutralizing anti-IL-12p40 mAb into PD- $1^{-1-}$ mice during DSS administration did not exacerbate colitis in PD-1 ${ }^{-1-}$ mice (Supplementary Fig. 1B). Although PD-1 has been demonstrated as an immuneinhibitory and anti-inflammatory molecule (13), our results indicate that PD-1 plays a pivotal role in the development of colitis, in which intestinal inflammation, unlike other autoimmune diseases, may be regulated by distinctive mechanisms, independently of augmented cytokine production.

\section{Co-housing PD-1-deficient mice with WT mice increases the susceptibility of DSS-induced colitis}

It was shown that mice lacking certain components of the immune system have altered gut microbiota that can be
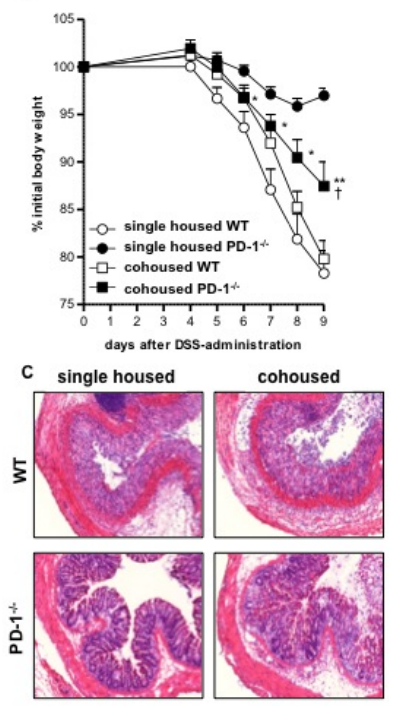
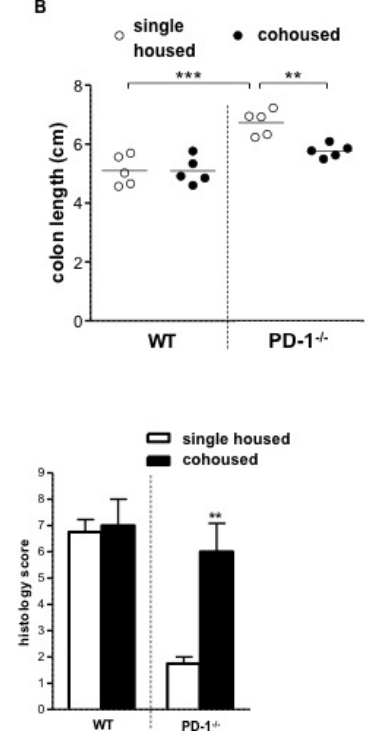

Fig. 2. Increased susceptibility to colitis of $\mathrm{PD}-1^{-1-}$ mice by cohousing with WT mice. WT and PD-1 ${ }^{-1-}$ mice were cohoused (co-house positive) for $4 \mathrm{wks}$, and then were treated with $2 \%$ DSS for $9 \mathrm{~d}$. (A) Change in body weight (\% of initial body weight, mean \pm SEM) during DSS-administration. Data are pooled from two independent experiments with similar results. ${ }^{*} \mathrm{P}<$ $0.05, * * \mathrm{P}<0.01$ vs. single housed controls and ${ }^{1} \mathrm{P}<0.05$ vs. WT, cohoused controls by Student's t-test. (B) Colon shortening of mice at $9 \mathrm{~d}$ after DSS-treatment. Each dot in the graph represents a single mouse. Data are representative of two independent experiments with similar results $* * P<0.01$, $* * * P<0.0001$ by Student's t-test. (C) Histology of colons at d 9. Histology score of DSS-treated WT $(n=4)$ and PD- $1^{-1-}$ mice $(n=6)$ were shown in the bar graph (mean \pm SEM). ${ }^{* * P}<0.01$, vs. single housed controls by Student's t-test. 
transmissible between mice and change susceptibility to intestinal inflammation (19-22). Kawamoto et al. showed that PD-1 ${ }^{-1-}$ mice exhibited alteration in their gut microbiota caused by impaired ability of follicular $T$ helper $\left(\mathrm{T}_{\mathrm{FH}}\right)$ cells to select proper IgA precursor cells in Peyer's patches in the absence of PD-1 (23). These studies led us to investigate whether the gut microbiota of $\mathrm{PD}-1^{-1-}$ mice contributes to their non-colitogenic phenotype. To this end, WT mice were cohoused with PD- $1^{-1-}$ mice at 1:1 ratio for 4 wks and then treated with DSS in drinking water for $9 \mathrm{~d}$. We found that PD-1 ${ }^{-1-}$ mice cohoused with WT mice had significant loss of body weight as compared to PD-1 ${ }^{-1-}$ mice housed alone, while the loss of body weight of WT mice was slightly ameliorated by cohousing with PD-1 ${ }^{-I-}$ mice, but was not statistically significant (Fig. 2A). In addition, cohoused PD-1 ${ }^{-1-}$ mice with WT mice showed colon shortening and extensive destruction of colon tissue, comparable to colitissusceptible WT mice (Fig. 2B, 2C). These results suggest that resistance to DSS-induced colitis in $\mathrm{PD}-1^{-/-}$mice is dependent on their gut microbiota that is less colitogenic than those in WT mice.

\section{Composition of gut microbiota is altered in PD-1-deficient mice}

Since our data in cohousing experiment supports the previous report showing altered composition of microbiota in $\mathrm{PD}-1^{-1-}$

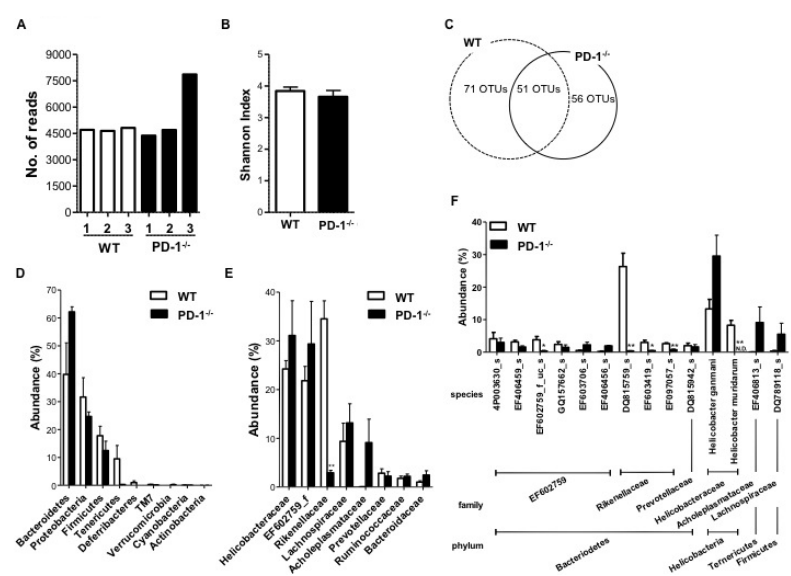

Fig. 3. Altered composition of colon microbial community in PD-1 ${ }^{-1}$ mice. Bacteria in pooled cecal contents of $\mathrm{WT}$ and $\mathrm{PD}^{-1-}$ mice were identified by $16 \mathrm{~S}$ rRNA gen-pyrosequencing analysis (A) Average sequences per sample. Each bar represents a single sample pooled from 2 or 3 mice. (B) Shannon Diversity Index (mean \pm SEM). (C) Venn diagram showing distributions of the shared OTUs. Taxon-based analysis at the level of (D) phylum, (E) family, and (F) species in bacterial composition between WT (white) and PD-1 ${ }^{-1-}$ mice (black) are shown as bar graphs. Data are pooled from three independent experiments, and each bar graph shows the abundance (\%, mean \pm SEM) in whole cecal bacteria. ${ }^{*} \mathrm{P}<0.05, * * P<0.01$ vs. WT controls by Student's t-test. mice (23), we next characterize microbial communities through $16 \mathrm{~S}$ rRNA gene-pyrosequencing analysis with cecal contents of $\mathrm{WT}$ or PD-1 ${ }^{-1-}$ mice. We acquired 31,103 sequences with an average $\sim 5,100$ reads/sample (Fig. 3A). A total of 178 operational taxonomic units (OTUs) was obtained at $97 \%$ sequence identity, and Shannon Diversity Index was calculated for each sample to estimate the diversity of fecal microbiota. The diversity index of PD-1 ${ }^{-1-}$ mice was similar to that of WT mice (Fig. 3B), but the composition of gut microbiota based on the OTU distribution differed significantly between these two groups (Fig. 3C). PD- $1^{-1-}$ mice shared 51 OTUs with WT mice, but had 56 unique OTUs. Taxon-based analysis indicated that microbiota in colon of WT and PD-1 ${ }^{-1-}$ mice was mainly composed of Bacteriodetes, Proteobacteria, Firmicutes, and Tenericutes (Fig. 3D). Interestingly, at the family level, Rikenellaceae was less abundant in PD-1 ${ }^{-1-}$ mice compared to WT mice (Fig. 3E). At the species level, 5 species in major species ( $>2 \%$ abundance) were significantly decreased in $\mathrm{PD}-1^{-1-}$ mice compared to WT mice, among which 3 unculturable species (DQ815759_s, EF603419_s, EF097057 s) belonged to Rikenelleceae family, and other species is Helicobacter muridarum that was not detected in PD-1 ${ }^{-1-}$ mice (Fig. 3F). It was reported that enriched Rikenellaceae in IL-22 $2^{-1-}$ mice or high-fat diet-fed mice is
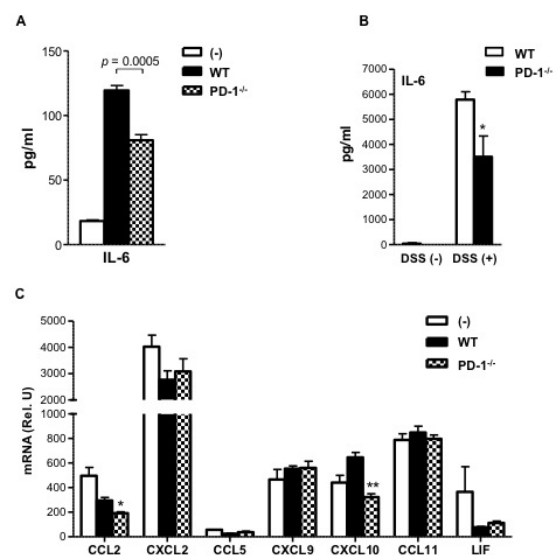

Fig. 4. Colon epithelial cells produce less inflammatory mediators in response to cecal contents of PD- $1^{-1-}$ mice. (A) $1 \times 10^{5}$ of CT-26 cells were treated with cecal extract (CE) of pooled naive $\mathrm{WT}(\mathrm{n}=4)$ or PD-1 ${ }^{-1-}$ mice $(\mathrm{n}=4)$ mice for $24 \mathrm{~h}$. Expression of IL-6 in culture supernatant was measured by ELISA. (B) Colon lamina propria mononuclear cells (LPMCs) from DSS-untreated (DSS negative) or DSS-treated (DSS positive) mice were isolated and stimulated in vitro with CpG DNA for $24 \mathrm{~h}$. IL-6 production in culture supernatant was measured by ELISA. The bar graph shows amount (mean \pm SEM) of indicated cytokines $(\mathrm{pg} / \mathrm{ml})$. Data are pooled from three different experiments $(n=4-7$ in each group). ${ }^{*} \mathrm{P}<0.05,{ }^{*} \mathrm{P}<0.01$ vs. WT controls by Student's t-test. (C) Relative mRNA level of chemokines was determined in CE-treated CT-26 cells, normalized by N32 expression. ${ }^{*} \mathrm{P}<0.05$, $* * \mathrm{P}<0.01$ vs. WT controls by Student's $t$-test. Representative data (mean \pm SEM) from two independent experiments are shown. 
associated with intestinal inflammation $(22,24)$, and monoassociation of $H$. muridarum in severe combined immunodeficiency (SCID) mice provoked intestinal inflammation in T cell transfer model of colitis $(25,26)$. Therefore, our results suggest that PD-1 deficiency resulted in altered composition of gut microbiota, which is associated with resistance to DSSinduced colitis.

Colon epithelial cells produce less inflammatory mediators in response to cecal extracts from PD-1-deficient mice To further determine whether colon microbiota of $\mathrm{PD}^{-1}{ }^{-1-}$ mice is less colitogenic than WT commensal bacteria, we stimulated CT-26 colon epithelial cell lines with cecal extracts from WT or PD- $1^{-1-}$ mice. IL-6 production was significantly reduced in colon epithelial cells when stimulated with cecal contents from PD-1 ${ }^{-1-}$ mice (Fig. 4A), which indicates direct influence of altered microbiota on the production of inflammatory cytokine in epithelial cells. Consistently, decrease in IL-6 production was also observed in colon LPMC of PD-1 ${ }^{-1-}$ mice after DSS treatment (Fig. 4B). Again, the altered microbiota may play a role, albeit we may not rule out the possibility that the absence of PD-1 in LPMC also contributes to the production of cytokine. Production of other inflammatory cytokines, including TNF- $\alpha$ and IL-1 $\beta$ was not significantly different between $\mathrm{WT}$ and $\mathrm{PD}-1^{-1-}$ mice (Supplementary Fig. 2). Chemokines work as inflammatory mediators in various diseases mainly by recruiting immune cells to the site of inflammation. During IBD progress, expression of chemokines and their receptors increased, which include monocyte chemotactic protein 1 (MCP-1, CCL2) and 3 (MCP-3), epithelial neutrophil activating protein 78 (CXCL5), macrophage inflammatory proteins $1 \alpha$ and $1 \beta$ (MIP- $1 \alpha$ and $-1 \beta$ ), interferon inducible protein 10 (IP-10, CXCL10), and fractalkine (CX3CL1) (27). Therefore, we analyzed an array of chemokines and found that expression of CCL2 and CXCL10 was significantly reduced in colon epithelial cells in response to cecal extracts from PD-1 ${ }^{-1-}$ mice (Fig. 4C), which are known to be over-expressed in the colon of ulcerative colitis patients and play a participatory role in infiltration of effector cells into the inflamed tissues $(28,29)$. Thus, these results suggest that altered microbiota composition confers protection of PD-1-deficient mice from DSS-induced colitis by decreasing the production of inflammatory mediators.

Role of intestinal microbiota in the pathogenesis of IBD has been highlighted for a recent decade. In this study, we reveal that PD- $1^{-1-}$ mice exhibit increased resistance to experimental colitis through altering composition of gut microbiota, which leads to reduction of inflammatory mediators in the colon. These findings are contrary to the well-known function of PD-1 in terms of regulating immune activation and inflammation. Based on our 16S rRNA analysis, this unexpected role of PD-1 in the DSS-induced colitis may be consequence of complicated interplay between the mucosal immune system, epithelium, and the microbiota in the intestine, in which PD-1 may exert its distinct effect on the development of colitis. Detailed mechanisms by which the altered microbiota in PD-1 ${ }^{-1-}$ mice modulates gut inflammation, with which types of immune cells are being targeted in the colon, remain to be further investigated. Moreover, our study also suggests that immune-modulatory activity via changing gut microbiota needs to be carefully considered, when we manipulate PD-1 signaling, e.g. anti-PD-1 mAb or PD-L1-Fc protein, in various pathophysiological situations, including inflammatory diseases and cancer.

\section{MATERIALS AND METHODS}

\section{Animal studies}

Specific-pathogen-free C57BL/6 (B6) mice were purchased from The Jackson Laboratory (Bar Harbor, ME). PD-1 ${ }^{-1-}$ mice in B6-background were obtained from Dr. Tasuku Honjo (Kyoto University, Japan) via Dr. Sang-Nae Cho (Yonsei University, Korea). Mice were bred and maintained in the animal facility at the Pohang University of Science and Technology Biotech Center (Pohang, Korea), and 6- to 10-wk-old mice were used for all experiments. To induce colitis, B6 or PD- ${ }^{-1-}$ mice were fed with $2 \%(\mathrm{w} / \mathrm{v})$ dextran sulfate sodium (DSS; m.w. of 36-50 kDa; MP Biomedicals, $\mathrm{OH}$ ) in drinking water for $9 \mathrm{~d}$. For cohousing experiment, female, 6-wk-old B6 and PD-1 KO mice were housed together at a ratio 1:1 for 4 wks prior to DSS administration.

\section{Histology}

Distal colon tissues were frozen sectioned and stained with H\&E. Histological damage was scored as described with minor modification (30).

\section{Preparation of lamina propria mononuclear cells (LPMCs)}

At $9 \mathrm{~d}$ after DSS-administration, LPMCs in colons were isolated as described (31). In Brief, isolated colons were incubated in HBSS with $5 \%$ FBS and 2 mM EDTA ( $5 \%$ HBSS + EDTA) and were digested in $5 \%$ HBSS with $1.5 \mathrm{mg} / \mathrm{ml}$ collagenase $\mathrm{D}$ and $0.5 \mathrm{mg} / \mathrm{ml}$ DNase I (both from Roche Diagnosis, Indianapolis, $\mathrm{IN})$ in $37^{\circ} \mathrm{C}$ for $40-50 \mathrm{~min}$.

\section{Preparation of cecal extracts}

For preparation of cecal extracts, frozen cecal contents from naïve $\mathrm{B} 6$ and $\mathrm{PD}-1^{-1-}$ mice were thawed and well suspended in 5 volumes of sterile water. After centrifugation (4000 rpm, $30 \mathrm{~min}$ ), supernatant werewas collected and filtered through $0.22 \mu \mathrm{m}$ filters.

\section{Analysis of inflammatory cytokine expression}

$1 \times 10^{6}$ cells $/ \mathrm{ml}$ of LPMCs were stimulated with $1 \mu \mathrm{M}$ CpG-DNA 1668 (Invitrogen, Carlsbad, CA) for $24 \mathrm{~h}$. In the other study, mouse epithelial cell line, CT-26, were cultured at $1 \times 10^{5}$ cells in $190 \mu$ of RPMI $+10 \%$ FBS with $10 \mu \mathrm{l}$ of cecal extracts for $24 \mathrm{~h}$. The amount of cytokines in culture 
supernatant was measured by ELISA.

\section{Meta 16S rRNA sequencing}

Bacterial genomic DNA in cecal contents was extracted with FastDNA SPIN kit for soil (MP Biomedicals, $\mathrm{OH}$ ) according to the manufacturer's protocol. PCR was performed with Taq DNA polymerase and primers to the $\mathrm{V} 1-\mathrm{V} 3$ region of the $16 \mathrm{~S}$ rRNA gene. Then, the amplified DNA was used as template for 454 GS Junior (Roche Diagnosis, Indianapolis, IN) pyrosequencing (outsourced in ChunLab, Inc. Korea). Filter-passed 3,000 reads were subjected to OTU (operational taxonomic unit) analysis with the cutoff similarity of $97 \%$ identity by using CLcommunity ${ }^{\mathrm{TM}}$ software.

\section{Quantitative real time PCR}

Total RNA from CT-26 cells was extracted by using ReliaPrep $^{\text {TM }}$ RNA Cell Miniprep System, and CDNA was synthesized by using GoScript ${ }^{\text {TM }}$ Reverse Transcription System (both from Promega, Fitchburg, WI). Quantitative Real-time PCR was performed with SYBR Green PCR Master Mix (Applied Biosystems, CA). The following primer sets were used: CCL2, 5'-GCTGGAGCATCCACGTGTT-3' and 5'-ATCTTGCTGGTGA ATGAGTAGCA-3'; CXCL2, 5'-CGCCCAGACAGAAGTCATAG$3^{\prime}$ and 5'-TCCTCCTTTCCAGGTCAGTTA-3'; CCL5, 5'-CACCA CTCCСТGCTGCTT-3' and 5'-ACACTTGGCGGTTCCTTC-3'; CXCL9, 5'-TTTTGGGCATCATCTTCCTGG-3' and 5'-GAGGTC TTTGAGGGATTTGTAGTGG-3'; CXCL10, 5'-CTTCTGAAAGG TGACCAGCC-3' and 5'-GTCGCACCTCCACATAGCTT-3'; CCL11, 5'-GGCTGACCTCAAACTCACAGAAA-3' and 5'-ACA TTCTGGCTTGGCATGGT-3'; LIF, 5'-ATGTGCGCCTAACATG ACAG-3' and 5'-TATGCGACCATCCGATACAG-3'; L32, 5'GAAACTGGCGGAAACCCA-3' and 5'-GGATCTGGCCCTTGA ACC-3'.

\section{Statistics}

Data are typically shown as mean \pm SEM. Differences between the groups were assessed using two-tailed Student's t-test. A P-value of $<0.05$ was considered statistically significant.

\section{ACKNOWLEDGEMENTS}

This study was supported by the Korean Health Technology R\&D Project funded by the Ministry of Health \& Welfare, Korea (HI14C-2171), World Class 300 Project funded by the Small and Medium Business Administration (SMBA, S2367890 (S2416714)), Korea, and a BK21 Plus grant funded by the Ministry of Education, Korea (10Z20130012243).

\section{CONFLICTS OF INTEREST}

The authors have no conflicting interests.

\section{REFERENCES}

1. Kamada, N, Seo SU, Chen GY and Nunez G (2013) Role of the gut microbiota in immunity and inflammatory disease. Nat Rev Immunol 13, 321-335

2. Belkaid $Y$ and Hand TW (2014) Role of the microbiota in immunity and inflammation. Cell 157, 121-141

3. Yang J-Y and Kweon M-N (2016) The gut microbiota: a key regulator of metabolic disease. BMB Rep 49, 536-541

4. Matsuoka K and Kanai T (2015) The gut microbiota and inflammatory bowel disease. Semin Immunopathol 37, 47-55

5. Maloy $\mathrm{KJ}$ and Powrie $\mathrm{F}$ (2011) Intestinal homeostasis and its breakdown in inflammatory bowel disease. Nature 474, 298-306

6. Frank DN, St Amand AL, Feldman RA, Boedeker EC, Harpaz N and Pace NR (2007) Molecular-phylogenetic characterization of microbial community imbalances in human inflammatory bowel diseases. Proc Natl Acd Sci U S A 104, 13780-13785

7. Tong M, Li X, Wegener P et al (2013) A modular organization of the human intestinal mucosal microbiota and its association with inflammatory bowel disease. PLoS One 8, e80702

8. Manichanh C, Rigottier-Gois L, Bonnaud E et al (2006) Reduced diversity of fecal microbiota in Crohn's disease revealed by a metagenomic approach. Gut 55, 205-211

9. Zenewicz LA, Antov A and Flavell RA (2009) CD4 T-cell differentiation and inflammatory bowel disease. Trends Mol Med 15, 199-207

10. Stallmach A, Hagel S and Bruns T (2010) Adverse effects of biologics used for treating IBD. Best Pract Res Clin Gastroenterol 24, 167-182

11. Carter L, Fouser LA, Jussif J et al (2002) PD-1:PD-L inhibitory pathway affects both $\mathrm{CD} 4(+)$ and $\mathrm{CD} 8(+) \mathrm{T}$ cells and is overcome by IL-2. Eur J Immunol 32, 634-643

12. Cho HY, Choi EK, Lee SW et al (2009) Programmed death-1 receptor negatively regulates LPS-mediated IL-12 production and differentiation of murine macrophage RAW264.7 cells. Immunolo Lett 127, 39-47

13. Francisco LM, Sage PT and Sharpe AH (2010) The PD-1 pathway in tolerance and autoimmunity. Immunol Rev 236, 219-242

14. Kanai T, Totsuka T, Uraushihara K et al (2003) Blockade of B7-H1 suppresses the development of chronic intestinal inflammation. J Immunol 171, 4156-4163

15. Scadiuzzi L, Ghosh K, Hofmeyer KA et al (2014) Tissue-expressed B7-H1 critically controls intestinal inflammation. Cell Rep 6, 625-632

16. Solomon S, Mansor S, Mallon P et al (2010) The dextran sulphate sodium (DSS) model of colitis: an overview. Comp Clin Pathol 19, 235-239

17. Yao S, Wang S, Zhu Y et al (2009) PD-1 on dendritic cells impedes innate immunity against bacterial infection. Blood 113, 5811-5818

18. Kim DJ, Kim KS, Song MY et al (2012) Delivery of IL-12p40 ameliorates DSS-induced colitis by suppressing IL-17A expression and inflammation in the intestinal mucosa. Clin Immunol 144, 190-199

19. Elinav E, Strowig T, Kau AL et al (2011) NLRP6 
inflammasome regulates colonic microbial ecology and risk for colitis. Cell 145, 745-757

20. Vijay-Kumar M, Aitken JD, Carvalho FA et al (2010) Metabolic syndrome and altered gut microbiota in mice lacking Toll-like receptor 5. Science 328, 228-231

21. Salzman NH, Hung K, Haribhai D et al (2010) Enteric defensins are essential regulators of intestinal microbial ecology. Nat Immunol 11, 76-83

22. Zenewics LA, Yin X, Wang G et al (2013) IL-22 deficiency alters colonic microbiota to be transmissible and colitogenic. J Immunol 190, 5306-5312

23. Kawamoto S, Tran TH, Maruya M et al (2012) The inhibitory receptor PD-1 regulates $\lg$ A selection and bacterial composition in the gut. Science 336, 485-489

24. Kim KA, Gu W, Lee IA et al (2012) High fat diet-induced gut microbiota exacerbates inflammation and obesity in mice via the TLR4 signaling pathway. PLoS One 7, e47713

25. Jiang $H Q$, Kushnir $N$, Thurnheer $M C$ et al (2002) Monoassociation of SCID mice with Helicobacter muridarum, but not four other enterics, provokes IBD upon receipt of T cells. Gastroenterology 122, 1346-1354

26. Dijkstra G, Yuvaraj S, Jian HQ et al (2007) Early bacterial dependent induction of inducible nitric oxide synthase (iNOS) in epithelial cells upon transfer of CD45RB(high) CD4(+) T cells in a model for experimental colitis. Inflamm Bowel Dis 13, 1467-1474

27. Danese S and Gasbarrini A (2005) Chemokines in inflammatory bowel disease. J Clin Pathol 58, 1025-1027

28. Uguccioni $M$, Cionchetti $P$, Robbiani DF et al (1999) Increased expression of IP-10, IL-8, MCP-1, and MCP-3 in ulcerative colitis. Am J Pathol 155, 331-336

29. Hyun JG, Lee G, Brown JB et al (2005) Anti-interferoninducible chemokine, CXCL10, reduces colitis by impairing $\mathrm{T}$ helper-1 induction and recruitment in mice. Inflamm Bowel Dis 11, 799-805

30. Seong MA Woo JK, Kang JH et al (2015) Oral administration of fermented wild ginseng ameliorates DSS-induced acute colitis by inhibiting NF-kB signaling and protects intestinal epithelial barrier. BMB Rep 48, 419-425

31. Geem D, Medina-Contreras O, Kim W, Huang CS and Denning TL (2012) Isolation and characterization of dendritic cells and macrophages from the mouse intestine. J Vis Exp e4040 